\title{
Differential microRNA expression analyses across two brain regions in Alzheimer's disease
}

\author{
Valerija Dobricic $^{1 *}$, Marcel Schilling ${ }^{1 *}$, Jessica Schulz ${ }^{1}$, Ling-Shuang Zhu ${ }^{2}$, Chao-Wen Zhou ${ }^{2}$, \\ Janina Fuß ${ }^{3}$, Sören Franzenburg ${ }^{3}$, Ling-Qiang Zhu ${ }^{2}$, Laura Parkkinen ${ }^{4}$, Christina M. Lill ${ }^{1,5}$, \\ Lars Bertram ${ }^{1,6}$
}

*These authors contributed equally

\section{Affiliations:}

1. Lübeck Interdisciplinary Platform for Genome Analytics (LIGA), University of Lübeck, Lübeck, Germany

2. Department of Pathophysiology, Key Lab of Neurological Disorder of Education Ministry, School of Basic Medicine, Tongji Medical College, Huazhong University of Science and Technology, Wuhan, China

3. Institute of Clinical Molecular Biology, Kiel University and University Medical Center Schleswig-Holstein, Kiel, Germany

4. Nuffield Department of Clinical Neurosciences, Oxford Parkinson's Disease Centre, University of Oxford, UK

5. Aging and Epidemiology Unit (AGE), School of Public Health, Imperial College London, London, UK

6. Center for Lifespan Changes in Brain and Cognition (LCBC), Department of Psychology, University of Oslo, Oslo, Norway

\section{Corresponding Author:}

Prof. Dr. Lars Bertram

Lübeck Interdisciplinary Platform for Genome Analytics (LIGA)

University of Lübeck

Ratzeburger Allee 160, Haus V50, 1st floor, Room 319

23562 Lübeck, Germany

Telephone: +4945131017490

Email: lars.bertram@uni-luebeck.de

Running title: Differential miRNA expression in AD brain

Keywords: Alzheimer's disease; RNA sequencing; miRNA; differential expression; brain; epigenetics 


\begin{abstract}
(247/250)
Background: Dysregulation of microRNAs (miRNAs) is involved in the pathogenesis of neurodegenerative diseases, including Alzheimer's disease (AD). Hitherto, sample sizes from differential miRNA expression studies in AD are exceedingly small aggravating any biological inference. To overcome this limitation, we investigated candidate miRNAs previously showing compelling evidence in the literature in a large collection of brain samples from AD and control individuals.
\end{abstract}

Methods: Brain tissue was derived from superior temporal gyrus (STG) and entorhinal cortex (EC) from $100 \mathrm{AD}$ patients and 99 control individuals. Expression of six selected miRNAs was assessed either by qPCR (STG) or small RNA sequencing (EC). Brain region-dependent differential miRNA expression was assessed in a transgenic AD mouse model. All results were combined with those from other datasets by meta-analysis.

Results: MiR-129-5p, miR-132-5p, and miR-138-5p were significantly downregulated in AD vs. controls both in STG and EC. In contrast, we found no strong evidence for differential miRNA expression for miR-125b-5p and miR-501-3p, which previously ranked high by meta-analysis. In addition, we observed miR-195-5p to be significantly upregulated in EC of AD, but not in STG. The brain region-specific nature of differential miR-195-5p expression was corroborated by targeted qPCR analyses in an AD transgenic mouse model.

Conclusions: Using two different methods (qPCR and small RNA-seq) in two separate brain regions in $~ 200 \mathrm{AD}$ patients and controls we more than doubled the available sample size for most miRNAs tested. Differential miRNA expression analyses confirm the likely involvement of miR-129-5p, miR-132-5p, miR-138-5p, and miR-195-5p in AD pathogenesis. 


\section{Introduction}

Alzheimer's disease (AD) is the most prevalent neurodegenerative disease characterized by progressive loss of memory and cognition eventually leading to dementia. While the pathogenic mechanisms underlying AD susceptibility are not yet completely understood, it is well established that susceptibility to AD is determined by the complex interplay of genetic, environmental, and epigenetic factors. High heritability estimates both for late (LOAD) and early (EOAD) onset AD support a crucial role of genetics, which reach beyond causal mutations. Namely, heritability for LOAD was estimated to be between $60-80 \%$ (1), and for EOAD as $>90 \%$ (2). In addition to genetic variants, epigenetic factors, e.g. DNA methylation and gene expression regulation by microRNAs (miRNAs), are increasingly recognized to play an important role in the etiology of $\mathrm{AD}(3,4)$.

MiRNAs are 18-25 nt long RNA molecules that bind to complementary sequence elements in the mRNA transcripts of protein coding genes to initiate transcript degradation or translational inhibition and thus repress protein synthesis $(5,6)$. Given their important role in the regulation of gene expression, miRNAs became a topic of many studies investigating their regulatory function, role as potential biomarkers, and/or therapeutic targets for a range of human disorders, including AD $(7,8)$. The situation is aggravated by technical factors such as the use of heterogeneous tissues for the analysis (e.g. different brain regions, different blood cells subpopulations), application of different methods for miRNA quantification and analysis, and use of small sample sizes. Over time, this has led to a vast body of - partially contradicting literature, which has become increasingly difficult to follow and interpret. To overcome these limitations, we recently conducted a systematic meta-analysis of differential miRNA expression 
studies in AD and identified 25 miRNAs showing study-wide significant differential expression in brains of $\mathrm{AD}$ cases vs. controls (9).

The goal of the present study was to assess the top-ranking meta-analytic differential miRNA expression results in a large and independent collection of brain samples from AD patients and controls. Specifically, we determined the expression levels of six miRNAs in two brain regions (entorhinal cortex [EC]) and superior temporal gyrus [STG]) collected from the same 200 individuals using either small RNA sequencing (EC) or TaqMan probe-based qPCR (STG). Moreover, we updated our previous meta-analyses with both the results from our current study and recent data from the literature. Brain region specific expression changes for one miRNA were assessed in different brain regions of an AD transgenic mouse model.

\section{Methods and Materials}

\section{Human samples}

Snap-frozen, postmortem human brain tissue from 100 AD patients and 99 elderly control individuals were obtained from the Oxford Brain Bank. These were derived from STG (Brodmann area BA21) and EC (Brodmann area BA28; for this region only $n=91$ AD and $n=92$ controls were available). The Ethics Committees of Oxford University and University of Lübeck approved the use of the human tissues for our study and all participants gave informed consent. The AD patients and healthy controls were part of the longitudinal, prospective Oxford Project to Investigate Memory and Aging (OPTIMA) using protocols which have been described in detail elsewhere (10). All subjects underwent a detailed clinical history, physical examination, assessment of cognitive function (Cambridge Examination of Mental Disorders of the Elderly 
(CAMDEX) (11) with the Cambridge Cognitive Examination (CAMCOG) and Mini-Mental

State Examination (MMSE) biannually. The pathological diagnosis of AD was made using the Consortium to Establish a Registry for Alzheimer's disease (CERAD)/National Institutes of Health (NIH) criteria and Braak staging (12-14). All included patients were of white European descent by self report.

\section{Selection of miRNAs for follow-up analysis}

In our recent systematic meta-analyses of differential miRNA expression studies in AD, 25 miRNAs showed study-wide ( $\alpha=1.08 \mathrm{E}-04)$ significant differential expression in brain (9). For

the present study, we selected those showing "strong evidence" for differential expression among the top 10 miRNAs. The term "strong evidence" refers to meta-analyses with $\geq 80 \%$ of included studies showing the same direction of effect. In total, six miRNAs were selected: miR-125b-5p, miR-129-5p, miR-132-5p, miR-138-5p, miR-195-5p, and miR-501-3p. In addition, we initially ran miR-423-5p and let-7b-5p alongside to serve as endogenous controls for the qPCR assays. This selection was based on recommendations of the TaqMan Advanced miRNA assay manufacturer, previous use in the literature, and low rank or absence among AD and PD brain miRNA differential expression meta-analysis results $(9,15)$. However, our novel small RNA sequencing analysis on the EC sections showed that miR-423-5p was modestly but significantly downregulated in AD cases as compared to controls, so we eventually only used let-7b-5p as endogenous control assay for qPCR.

MiRNA isolation and quantitative PCR in superior temporal gyrus (STG) sections 
For the 199 STG samples, total RNA, including miRNA, was extracted from approximately $25 \mathrm{mg}$ of brain sections using the mirVana miRNA kit (Thermo Fisher Scientific, USA) following the manufacturer's instructions. Immediately after extraction, RNA samples were treated with DNase (TURBO DNA-free kit, Thermo Fisher Scientific). Total RNA concentration and purity were measured using a NanoDrop 2000 instrument (Thermo Fisher Scientific). Further, RNA integrity was assessed using a Bioanalyzer 2100 instrument in conjunction with the RNA 6000 Nano LabChip kit (Agilent Technologies, USA).

Reverse transcription of total miRNA was carried out with the TaqMan Advanced miRNA cDNA Synthesis Kit (Thermo Fisher Scientific) using an input of $10 \mathrm{ng}$ total RNA, according to manufacturer's instructions. Quantitative assessment of the expression of miR125b-5p (MIMAT0000423), miR-129-5p (MIMAT0000242), miR-132-5p (MIMAT0004594), miR-138-5p (MIMAT0000430), miR-195-5p (MIMAT0000461), and miR-501-3p (MIMAT0004774), along with two endogenous control miRNAs (miR-423-5p and let-7b-5p, although only the latter was used in this function, see above) was performed in 384-well format using TaqMan pre-spotted assays (Thermo Fisher Scientific) on a QuantStudio-12K-Flex system. Samples were assayed in triplicates. In order to minimize potential batch effects, cases and controls were randomly distributed across plates. Raw data analysis was performed using ExpressionSuite Software v1.2 (Thermo Fisher Scientific). Replicates with differences in Ct value $>0.5$ were excluded from subsequent analysis. All other resulting $\mathrm{Ct}$ values were used in the downstream differential miRNA expression analyses (see below).

Small RNA sequencing in entorhinal cortex $(E C)$ 
EC sections were available for a subset of 183 individuals (91 cases, 92 controls) included in our current study. Quantification of the expression of the six miRNAs of interest was based on small RNA sequencing which was performed as part of another ongoing project. To this end, total RNA, including miRNA, was purified and quantified using the same methods as described above. Library preparation and subsequent sequencing were conducted at the NGS Competence Centre at IKMB institute (Kiel, Germany). Libraries were prepared using the NextFlex Small RNA-Seq kit (PerkinElmer, USA), according to manufacturer's instructions, and sequenced on a HiSeq 4000 instrument (Illumina, USA) with 1x50bp reads. Sequencing adapters were trimmed from raw reads using Flexbar v3.4.0 (16,17). Reads were mapped and miRNAs quantified against miRBase v22.1 $(18,19)$. Normalization and differential expression analysis was carried out using the R packages DESeq2 v1.28.1 (20) and apeglm v1.12.0 (for LFC shrinkage) (21).

\section{Statistical analysis}

(Log) normalized sRNA-seq counts and $\Delta \mathrm{Ct}$ values, respectively, were averaged over replicates and scaled by the corresponding value for let-7b-5p (endogenous control). These values $\left(\Delta \Delta \mathrm{Ct}\right.$, in the case of qPCR) were transformed to relative quantity measures $\left(2^{\Delta \Delta \mathrm{Ct}}\right)$ and compared across conditions (AD cases vs. controls). Additionally, per miRNA and method (qPCR, sRNA-seq), a (Gaussian) generalized linear model (GLM) was trained to predict the (scaled and centered) abundance measures from case-control status and the following (scaled and centered, if applicable) potential confounding variables: age at death, sex, RNA integrity (RIN), post-mortem interval. The F-statistic was used to assess the significance of the effect estimate of 
the AD case-control status on the expression readout. Analogously, we utilized all samples with Braak staging information to train GLMs predicting gene expression based on Braak stage. To this end, the corresponding binary (AD case vs. control) variable was replaced by a continuous (scaled and centered) Braak stage value. All other analysis steps and parameters (confounding variables) were identical to the case vs. control analyses.

Multiple testing correction was performed using Bonferroni's method adjusting for 6 independent miRNAs, resulting in a one-sided study-wide $\alpha$ of $0.0167(=2 *(0.05 / 6))$. One-sided testing is applicable here given the specific hypotheses tested based on prior evidence from Takousis et al (9).

\section{Literature search and meta-analyses}

To search for novel papers on the investigated miRNAs published since the data freeze in Takousis et al. (9) we performed another systematic PubMed search (www.ncbi.nlm.nih.gov/pubmed/) using the search term from the original paper "(microRNA OR miRNA OR miR OR micro-RNA) AND Alzheimer*”. We included articles published until January 31, 2021, in peer-reviewed journals in English. Citations were assessed for eligibility using the title, abstract or the full text, as necessary. Data was extracted from studies comparing the brain expression in samples of AD patients versus controls for any of the six miRNAs analyzed here.

To arrive at summary estimates for the overall evidence of differential expression for the six tested miRNAs, we combined all data using the same meta-analysis workflow and methods as described previously (9). Data included were 1) database assembled for Takousis et al., 2) novel data generated in our brain samples, and 3) novel publications on these miRNAs identified 
in our literature search. P-values computed for the meta-analyses represent two-sided tests, as no specific hypothesis on the effect direction could be made. To correct for multiple testing, we used the same conservative threshold as in our original publication, i.e. and $\alpha$ of $1.08 \mathrm{E}-04$ reflecting the 461 miRNAs tested in that study (9).

\section{Animal work}

All animal experiments were performed according to the "Policies on the Use of Animals and Humans in Neuroscience Research" revised and approved by the Society for Neuroscience in 1995. The conduct of all animal experiments was approved by the Animal Ethics Committee of Huazhong University of Science and Technology.

To confirm the region-specific differential miRNA expression of mir-195-5p observed in the human samples, we also measured brain expression patterns of miR-195-5p in EC, hippocampus and temporal cortex in two different AD mouse models (P301S and APP/PS1) through qPCR and fluorescence in situ hybridization (FISH). APP/PS1 mice (\#34829) and P301S mice (\#008169) were purchased from Jackson laboratory, and wild type littermates were used as control. Mice were sacrificed at 6 months of age and total RNA, including miRNA, was extracted from brain sections of control and AD mice, and reverse transcription of total miRNA was carried out as described above. After the PCR reaction, amplified DNA fragments were verified by gel electrophoresis on a $3 \%$ agarose gel. Amplification and analysis were performed in the iCycler iQ Multicolor Real-Time PCR Detection System (BioRad, USA).

After loading on HistoBond Slides (VWR, USA) and drying overnight at $42^{\circ} \mathrm{C}, 30 \mu \mathrm{m}$ brain slices were incubated in protease $\mathrm{K}$ detergent at $37^{\circ} \mathrm{C}$ for $30 \mathrm{~min}$, and then transferred to $0.25 \%$ acetic anhydride and $0.1 \mathrm{M}$ triethanolamine for $10 \mathrm{~min}$. Hybridization buffers containing 
5' HEX- labeled probes were used to incubate slices in an in-situ hybridization apparatus (Boekel Slide Moat, USA) at $52{ }^{\circ} \mathrm{C}$ under the condition of heat preservation and humidification. Subsequently, brain slices were incubated with anti-Calbindin D-28k (Swant, \#300, diluted in 1:500) and Alexa Flour 546 (ThermoFisher, diluted in 1:200). The images were collected using a confocal laser scanning microscope (Zeiss Axio Imager Z2, Maerzhaeuser Motorized Scanning Stage), and analyzed using Zen Pro. Probes were designed and purchased from Tsingke, China.

\section{Results}

Demographics and RNA quality assessments

The average age of death was 81.6 years (median $=83$ years, interquartile range $[\mathrm{IQR}]=$ 9.25 years) for $\mathrm{AD}$ patients and 78 years (median $=82$ years, $\mathrm{IQR}=20$ years) for controls (Welch t-test, $\mathrm{P}=0.024)$. The average postmortem interval was $57.07 \mathrm{~h}($ median $=48 \mathrm{~h}, \mathrm{IQR}=$ 43.75h) for $\mathrm{AD}$ cases, and 50.23h (median $=48 \mathrm{~h}, \mathrm{IQR}=30 \mathrm{~h})$ for controls (Welch t-test, $\mathrm{P}=$ 0.128) (Table 1). There was no significant difference in the sex distribution between the AD and the control group (chi-square test, $\mathrm{P}=0.353$ ). Detailed distributions of Braak stages are given in Table 1. Numbers above are for the larger STG dataset $(n=199)$ but were largely equivalent for the EC dataset $(n=183)$.

RNA yield was good across all STG (EC) samples with $490 \mathrm{ng}(650 \mathrm{ng})$ total RNA per mg of tissue weight. RIN values ranged between 1.2 (1.2) and 7.8 (6.3), with an average of 3.6 (3.0). Comparison of raw expression data showed that the distribution of $\mathrm{Ct}$ values (across all miRNA assays per sample) was similar for samples with lower $(\mathrm{RIN}<5)$ vs. higher RIN values $(\mathrm{RIN} \geq 5)$ (Supplementary Figure 1). The same was observed for the distribution of average $\mathrm{Ct}$ 
values for endogenous control assays in the samples with lower $(\mathrm{RIN}<5)$ and higher RIN values $(\mathrm{RIN}>5)\left(\right.$ mean $\mathrm{Ct}_{\mathrm{RIN}<5}=23.92$ vs. mean $\left.\mathrm{Ct}_{\mathrm{RIN} \geq 5}=23.99\right)$, indicating that miRNAs were not majorly affected by RNA degradation (data not shown), in line with Lau et al. (22).

Notwithstanding, we adjusted for differences in average RIN values in AD samples vs. controls $\left(\mathrm{RIN}_{\mathrm{AD}}=3.02\right.$ vs. $\mathrm{RIN}_{\mathrm{ctrl}}=4.2$; $\mathrm{t}$-test, $\left.\mathrm{P}<0.001\right)$ in the regression models to account for residual confounding. Visual inspection of the distribution of $\mathrm{Ct}$ values for endogenous control assays per sample revealed one outlier sample from the control group that was excluded from further analyses, so we continued with 100 AD cases and 98 controls.

\section{Differential miRNA expression analysis in brains of AD cases and controls}

Our qPCR-based expression analyses showed that three (i.e. miR-132-5p [P $=6.90 \mathrm{E}-22]$, miR-138-5p [P = 1.40E-04], miR-129-5p [P=6.00E-09]) of the six tested miRNAs, showed evidence for significant differential expression in STG sections in AD cases vs. controls (Table 2). All three miRNAs showed decreased expression levels in AD cases. Likewise, in EC, a significant downregulation of the same three miRNAs in AD patients was observed using a different experimental method (i.e. small RNA sequencing). In addition, we observed miR-195$5 p$ to be significantly upregulated in EC of AD vs. controls $(\mathrm{P}=0.00014)$, but not in $\mathrm{STG}(\mathrm{P}=$ 0.14). We observed no evidence for differential expression for neither of the other two tested miRNAs in either STG or in EC (Figure 1, Table 2). Overall, with the exception of miR-125b$5 p$, the direction of expression change was concordant in STG vs. EC for all tested miRNAs (Table 2).

Using Braak-staging as diagnostic variable yielded similar results to those obtained in the case vs. control analyses (Supplementary Table 1). In STG, these highlighted miR-129-5p, miR- 
138-5p, and miR-132-5p as differentially expressed. In EC, these analyses revealed the same three miRNAs and miR-195-5p.

\section{Meta-analysis of novel differential miRNA expression results with published evidence}

To assess the overall evidence of differential expression of the six miRNAs tested here we updated our earlier meta-analyses by combining 1) results from the database of Takousis et al., 2) STG-based results from the current study, and 3) data from additional studies testing any of the six miRNAs for differential miRNA expression in human brain samples not included in our previous report (only one such paper, on miR-129-5p, was identified (23)).

All meta-analysis results are shown in Table 2. There are at least three noteworthy observations to be made from these results: First, for all three miRNAs significant in our own brain dataset (i.e. miR-132-5p, miR-138-5p, and miR-129-5p; see above), the statistical evidence increased by several orders of magnitude (3-20x, as judged by P-value) upon meta-analysis, minimally reaching 3.63E-10 (for miR-195-5p). Second, in contrast to these strengthened results, two miRNAs that ranked very high in our previous assessment of the literature (9), i.e. miR125b-5p and miR-501-3p, were not replicated in our independent brain dataset and, as a result, now only show vastly reduced evidence of differential expression upon meta-analysis. Third, for one miRNA (i.e. miR-195-5p) we observed region-specific differences in differential expression (significant only in EC; see above). As a result, only the meta-analyses using data from EC ( $p=3.63 \mathrm{E}-10$; Supplementary Table 1) improved with respect to those from our previous study $(p=3.74 \mathrm{E}-07$; (ref. 9)). Taken together, our novel differential miRNA expression results derived from a new comparatively large dataset analyzed in combination with previously published data 
now nominate a revised and partially different set of miRNAs to be strongly linked to AD in brain.

Assessment of brain region-specific expression changes for miR-195-5p in two different AD mouse models

We further examined the expression of miR-195-5p in different brain regions of 6-monthold APP/PS1 and P301S mice. Consistent with the results observed in the human samples, we observed an upregulation of miR-195-5p in EC of P301S mice vs controls ( $p=0.0226)$, but not in hippocampus and temporal cortex (Figure 2). No differences were found in any of the analyzed brain regions of APP/PS1 mice vs. controls, indicating that miR-195-5p upregulation might be related to tau pathology, for which P301S is a model. Furthermore, FISH analysis showed that miR-195-5p is mainly increased in EC layer II Calb+ neurons, the most vulnerable neurons in the early stage of AD (22), in P301S but not APP/PS1 mice (Figure 2).

\section{Discussion}

In this study, we performed independent assessments of the most compelling miRNAs previously reported to show differential expression in brain samples of AD patients vs. controls. Specifically, we analyzed the expression of six "top" miRNAs from these meta-analyses in two brain regions in a comparatively large (n 200) dataset using two experimental methods of miRNA quantification. The results showed evidence for significant differential expression for three out of six of these miRNAs in STG and four out of six in EC. One AD miRNA (miR-195- 
5p) showed brain region-specific differential miRNA expression, i.e. only in EC but not STG in the same individuals. This latter finding was corroborated in an AD transgenic mouse model. Lastly, we updated our previous meta-analyses with the novel data generated here and by other groups on these miRNAs. The results now nominate a partially different set of miRNAs to be linked to $\mathrm{AD}$ in brain and reveal that miR-125-5p and miR-501-3p no longer range among the top-ranked miRNAs to show differential expression in AD brains.

Our novel results are noteworthy for several reasons. First, by analyzing brain sections from nearly 200 individuals, our study vastly increases the total available sample size for all six miRNAs tested. This is important given the observation that the median sample size of miRNA differential expression studies in brain in AD was only 42.5 (inter quartile range [IQR] 23-85) (9) prior to this study. Second, by analyzing two different brain regions (EC and STG) using two different experimental methods (qPCR and small-RNA sequencing) our results are relatively well protected against tissue- or methods-related artifacts. This is further evidenced by the fact that most (but not all, see below) differential miRNA expression results correspond well to the prior evidence. Third, by updating the previous meta-analyses from Takousis et $a l$. with both our novel data as well as other data from study published since our original assessment, the results provided herein represent the most current snapshot of miRNA expression data in the field. Specifically, the new meta-analyses considerably strengthened the evidence for four of the six tested miRNAs, i.e. miR-129-5p, miR-132-5p, miR-138-5p, and miR-195-5p. Interestingly, for the latter, we only observed significant differential expression in EC but not in STG in both human and mouse data, arguing for the need to analyze multiple brain regions in future studies. Finally, two miRNAs previously showing very strong evidence for differential expression in AD, i.e., miR-125b-5p and miR-501-3p, are no longer on the top of the list. Their drastic drop in 
significance (and perhaps functional importance) is the result of the size of our dataset, which exceeds the previously published sample sizes for these miRNAs by 2.5 - and 3.5-fold, respectively. While it remains possible that the non-validation in our dataset reflects a falsenegative finding, this appears unlikely given the consistency of our null findings across both brain regions and both molecular methods used. Hence, our data suggest that miR-125b-5p and miR-501-3p may be less relevant in AD pathogenesis than previously thought.

Despite its strengths, our study may also be subject to a number of limitations. First, while our sample size (n 200) was large compared to previous studies on the topic (medium sample size $=42.5$; (ref. 9)), it may have still been too small to detect minor differences in miRNA expression, so that all or some of our null findings may reflect false negatives. Second, with an average of 3.6 the RIN values of our samples was comparatively low, which may have led to both false positive as well as false-negative results. However, we went to great lengths at accounting for this limitation in our analyses (see methods and results) and found no evidence that low RIN values actually skewed our differential miRNA expression results. Moreover, there are multiple studies reporting that RIN values only had a negligible or no effect on the detection of miRNAs, unlike mRNAs which tend to gradually degrade with decreasing RIN values (25). In addition, the fact that most of the previous "top" miRNAs actually do show independent replication here also argues against a major impact of low RIN on our study. Third, despite being comparatively comprehensive in both size and scope, our study used RNA extracts from "bulk" brain sections. These comprise a mixture of different cell-types (e.g. neurons, immune cells) which may have confounded some of our results. The only bona fide remedy against this potential confounding would be to perform single-cell/single-nucleus RNA sequencing. 
However, this methodology is currently still comparatively expensive precluding analyses in sample sizes such as achieved here in the foreseeable future.

Since our study followed-up on previous work, the potential functional implications of the miRNAs highlighted to show consistent and highly significant differential expression here have not changed much and we refer to the discussion of Takousis et al. for more details. The most interesting aspect in this context is probably the assessment of whether or not the four validated miRNAs of this study target any of the known AD genes as judged by the 2013 GWAS from the IGAP (26). In the Takousis et al. report this had revealed a total of seven AD genes for the four miRNAs validated in our study, i.e. ADAMTS4 (miR-129-5p), APP (miR-138-5p and miR-195-5p), CD2AP (miR-195-5p), CNTNAP2 (miR-195-5p), and FERMT2 (miR-138-5p). Comparing the same target predictions to an updated list of AD genes identified by from two more recent GWAS $(27,28)$, as summarized in Bertram \& Tanzi (29) did not change these predictions. However, using an extended and even more recent list of GWAS results from the European Alzheimer's disease DNA biobank (EADB) project published as preprint (30) reveals several new connections, i.e. for ADAM17 \& USP6NL (both miR-129-5p), CTSB \& EED (miR138-5p), and ANK3 \& PLEKHA1 (miR-195-5p). Collectively, these results offer a direct link between two different molecular layers both showing an involvement in AD pathogenesis using entirely different methodologies. As such, they provide some first functional leads on the potential mechanisms by which the miRNAs found to be differentially expressed in our and previous work may unfold their effects. Future work is needed to validate and extend these potential functional implications.

In conclusion, by studying the expression patterns of six previously top-ranked miRNAs across two human brain regions in a sample of 200 AD patients and control individuals, we 
confirm the likely involvement of miR-129-5p, miR-132-5p, miR-138-5p, and miR-195-5p in AD pathogenesis.

\section{Acknowledgements}

This work was supported by the Deutsche Forschungsgemeinschaft (DFG) and the National Science Foundation China (NSFC) as a Joint Sino-German research project ("MiRNet-AD”, \#391523883) to L.B. and L.-Q.Z. Additional support was provided by the DFG Research Infrastructure NGS_CC (project 407495230) as part of the Next Generation Sequencing Competence Network (\#423957469). NGS analyses were carried out at the Competence Centre for Genomic Analysis (Kiel). We acknowledge the Oxford Brain Bank, supported by the Medical Research Council (MRC), Brains for Dementia Research (BDR) (Alzheimer Society and Alzheimer Research UK), Autistica UK and the NIHR Oxford Biomedical Research Centre.

\section{Disclosures}

Valerija Dobricic: Nothing to disclose.

Marcel Schilling: Nothing to disclose.

Jessica Schulz: Nothing to disclose.

Ling-Shuang Zhu: Nothing to disclose.

Chao-Wen Zhou: Nothing to disclose.

Janina Fuß: Nothing to disclose.

Sören Franzenburg: Nothing to disclose.

Ling-Qiang Zhu: Nothing to disclose.

Laura Parkkinen: Nothing to disclose. 
bioRxiv preprint doi: https://doi.org/10.1101/2021.05.31.446406; this version posted May 31, 2021. The copyright holder for this preprint (which was not certified by peer review) is the author/funder. All rights reserved. No reuse allowed without permission.

Christina M. Lill: Nothing to disclose.

Lars Bertram: Nothing to disclose. 


\section{References:}

1. Gatz M, Reynolds CA, Fratiglioni L, Johansson B, Mortimer JA, Berg S, et al. Role of genes and environments for explaining Alzheimer disease. Arch Gen Psychiatry. 2006;63(2):168-74.

2. Wingo TS, Lah JJ, Levey AI, Cutler DJ. Autosomal recessive causes likely in early-onset Alzheimer disease. Arch Neurol [Internet]. 2012 Jan 1;69(1):59-64. Available from: http://archneur.jamanetwork.com/article.aspx?doi=10.1001/archneurol.2011.221

3. Hébert SS, De Strooper B. Alterations of the microRNA network cause neurodegenerative disease. Trends Neurosci. 2009;32(4):199-206.

4. Silvestro S, Bramanti P, Mazzon E. Role of miRNAs in alzheimer's disease and possible fields of application. Int J Mol Sci. 2019;20(16):1-26.

5. Guo H, Ingolia NT, Weissman JS, Bartel DP. Mammalian microRNAs predominantly act to decrease target mRNA levels. Nature. 2010 Aug 12;466(7308):835-40. Available from: http://www.ncbi.nlm.nih.gov/pubmed/20703300

6. Bartel DP. MicroRNAs: Genomics, Biogenesis, Mechanism, and Function. Cell. 2004;116:28197.

7. Angelucci F, Cechova K, Valis M, Kuca K, Zhang B, Hort J. MicroRNAs in Alzheimer's disease: Diagnostic markers or therapeutic agents? Front Pharmacol. 2019;10(JUN):1-9.

8. Ludwig N, Fehlmann T, Kern F, Gogol M, Maetzler W, Deutscher S, et al. Machine Learning to Detect Alzheimer's Disease from Circulating Non-coding RNAs. Genomics, Proteomics Bioinforma. 2019;17(4):430-40. Available from: https://doi.org/10.1016/j.gpb.2019.09.004

9. Takousis P, Sadlon A, Schulz J, Wohlers I, Dobricic V, Middleton L, et al. Differential expression of microRNAs in Alzheimer's disease brain, blood, and cerebrospinal fluid. Alzheimer's Dement. 2019;15(11):1468-77.

10. Clarke R, Smith AD, Jobst KA, Refsum H, Sutton L, Ueland PM. Folate, vitamin B12, and serum total homocysteine levels in confirmed Alzheimer disease. Arch Neurol. 1998;55(11):1449-55. Available from: https://pubmed.ncbi.nlm.nih.gov/9823829/ 
11. Roth M, Tym E, Mountjoy CQ, Huppert FA, Hendrie H, Verma S, et al. CAMDEX. A standardised instrument for the diagnosis of mental disorder in the elderly with special reference to the early detection of dementia. Br J Psychiatry. 1986;149(DEC.):698-709. Available from: https://www.cambridge.org/core/journals/the-british-journal-of-psychiatry/article/abs/camdex-astandardised-instrument-for-the-diagnosis-of-mental-disorder-in-the-elderly-with-specialreference-to-the-early-detection-of-dementia/195948C408E197A5253CB3EE7EF6DFA0

12. Mirra S, Heyman A, McKeel D, SM S, Crain B, Brownlee L, et al. The Consortium to Establish a Registry for Alzheimer's Disease (CERAD). Part II. Standardization of the neuropathologic assessment of Alzheimer's disease. Neurology. 1991;41(4):479-86.

13. Hyman BT, Phelps CH, Beach TG, Bigio EH, Cairns NJ, Carrillo MC, et al. National Institute on Aging-Alzheimer's Association guidelines for the neuropathologic assessment of Alzheimer's disease. Alzheimer's Dement. 2012;8(1):1-13.

14. Braak H, Braak E. Neuropathological stageing of Alzheimer-related changes. Acta Neuropathol. 1991;82(4):239-59.

15. Schulz J, Takousis P, Wohlers I, Itua IOG, Dobricic V, Rücker G, et al. Meta-analyses identify differentially expressed micrornas in Parkinson's disease. Ann Neurol. 2019;85(6).

16. Dodt M, Roehr JT, Ahmed R, Dieterich C. FLEXBAR-flexible barcode and adapter processing for next-generation sequencing platforms. Biology (Basel). 2012;1(3):895-905.

17. Roehr JT, Dieterich C, Reinert K. Flexbar 3.0 - SIMD and multicore parallelization. Bioinformatics. 2017;33(18):2941-2.

18. Griffiths-Jones S, Grocock RJ, van Dongen S, Bateman A, Enright AJ. miRBase: microRNA sequences, targets and gene nomenclature. Nucleic Acids Res. 2006;34(Database issue):140-4.

19. Kozomara A, Birgaoanu M, Griffiths-Jones S. MiRBase: From microRNA sequences to function. Nucleic Acids Res. 2019;47(D1):D155-62.

20. Love MI, Huber W, Anders S. Moderated estimation of fold change and dispersion for RNA-seq data with DESeq2. Genome Biol. 2014;15(12):1-21. 
21. Zhu A, Ibrahim JG, Love MI. Heavy-Tailed prior distributions for sequence count data: Removing the noise and preserving large differences. Bioinformatics. 2019;35(12):2084-92.

22. Lau P, Bossers K, Janky R, Salta E, Frigerio CS, Barbash S, et al. Alteration of the microRNA network during the progression of Alzheimer's disease. EMBO Mol Med. 2013;5(10):1613-34.

23. Patrick E, Rajagopal S, Wong HKA, McCabe C, Xu J, Tang A, et al. Dissecting the role of noncoding RNAs in the accumulation of amyloid and tau neuropathologies in Alzheimer's disease. Mol Neurodegener. 2017;12(1):1-13.

24. Yassa MA. Ground zero in Alzheimer's disease. Nat Neurosci. 2014;17(2):146-7. Available from: http://dx.doi.org/10.1038/nn.3631

25. Jung M, Schaefer A, Steiner I, Kempkensteffen C, Stephan C, Erbersdobler A, et al. Robust MicroRNA stability in degraded RNA preparations from human tissue and cell samples. Clin Chem. 2010;56(6):998-1006.

26. Lambert JC, Ibrahim-Verbaas CA, Harold D, Naj AC, Sims R, Bellenguez C, et al. Meta-analysis of 74,046 individuals identifies 11 new susceptibility loci for Alzheimer's disease. Nat Genet. 2013;45(12):1452-8.

27. Jansen I, Savage J, Watanabe K, Bryois J, Williams D, Steinberg S, et al. Genome-wide metaanalysis identifies new loci and functional pathways influencing Alzheimer's disease risk. Nat Genet. 2019;51(3):404-13.

28. Kunkle BW, Grenier-Boley B, Sims R, Bis JC, Damotte V, Naj AC, et al. Genetic meta-analysis of diagnosed Alzheimer's disease identifies new risk loci and implicates $A \beta$, tau, immunity and lipid processing. Nat Genet. 2019;51(3):414-30.

29. Bertram L, Tanzi RE. Genomic mechanisms in Alzheimer's disease. Brain Pathol. 2020;30(5):966-77.

30. Bellenguez C, Küçükali F, Jansen I, Andrade V, Moreno-grau S. New insights on the genetic etiology of Alzheimer' s and related dementia. 2020;1-35. 


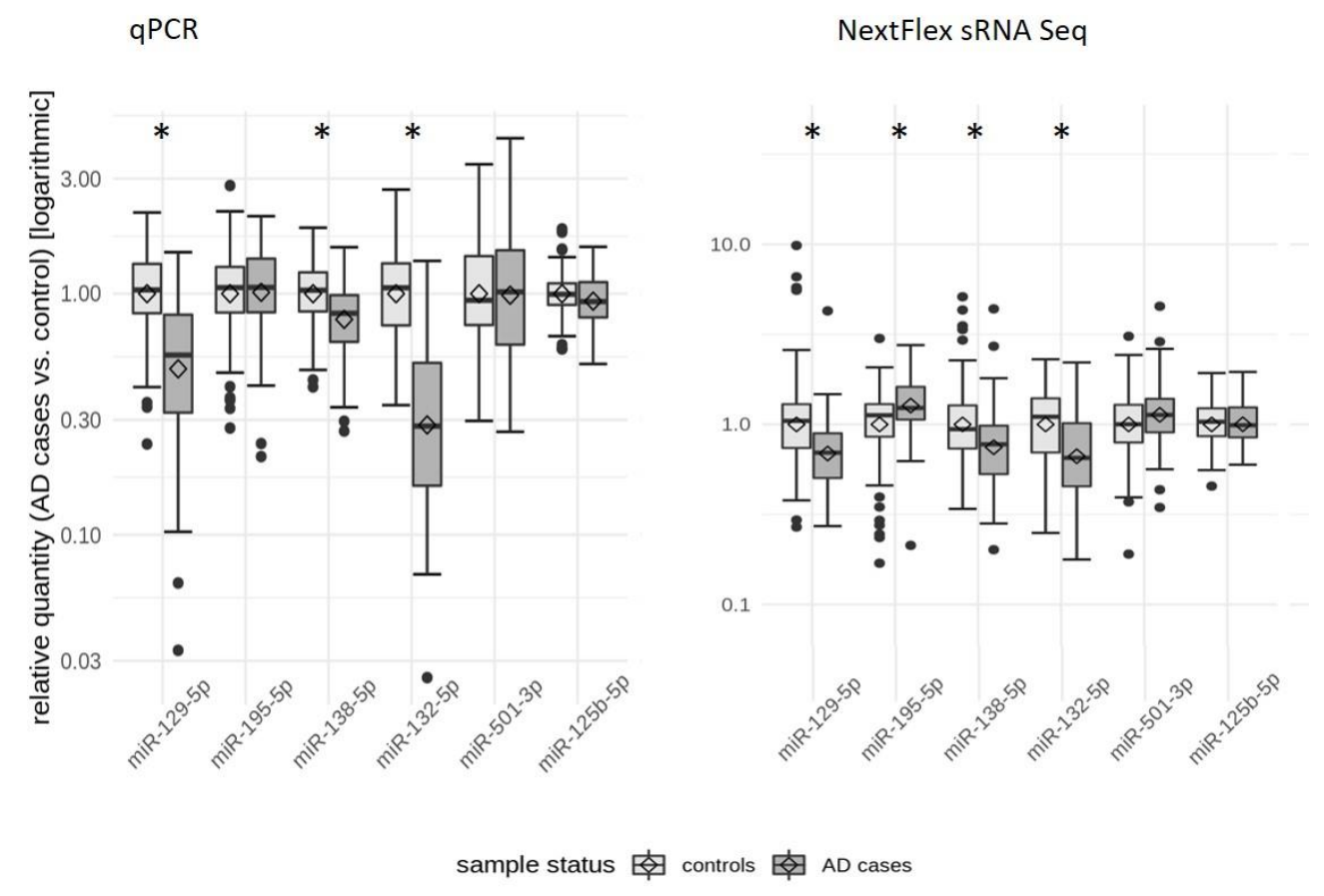

Figure 1. Expression levels of analyzed miRNAs in Alzheimer's disease patients relative to controls. Left plot: qPCR results, superior temporal gyrus samples; Right plot: miRNA sequencing results, entorhinal cortex samples; Bars filled in light grey - controls; Bars filled in dark grey - AD cases; $*$ - statistically significant difference at $\alpha=0.0167$ (see Methods). 
(a)

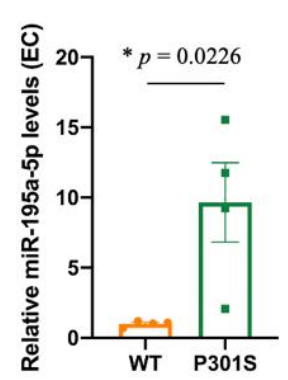

(e)

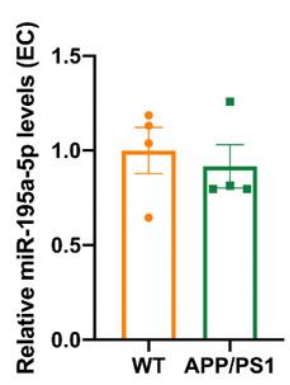

(b)

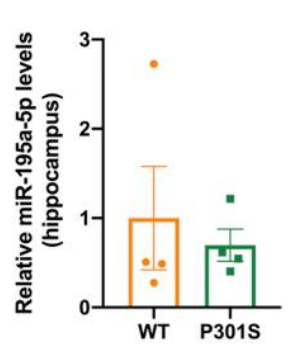

(f)

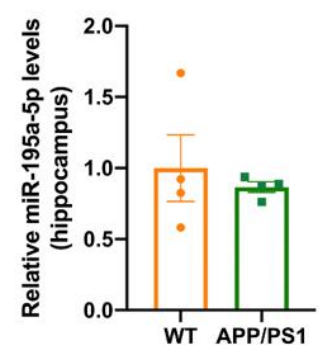

(c)

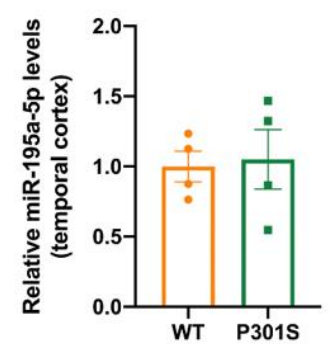

(g)

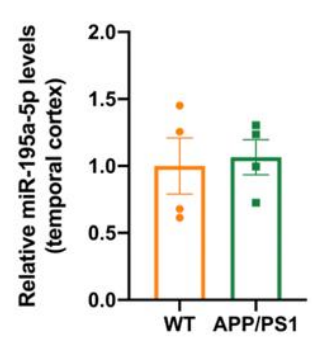

(d)

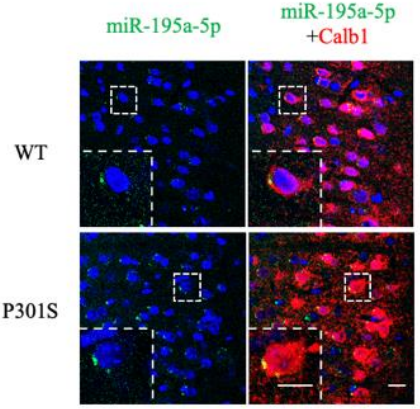

(h)

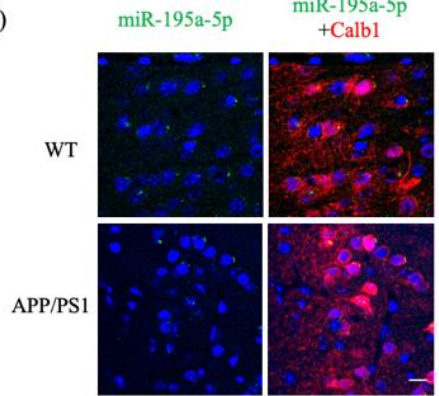

Figure 2. miR-195a-5p is increased in EC of P301S but not of APP/PS1 mice.

(a) Expression of miR-195a-5p in entorhinal cortex of 6-month-old P301S mice compared to wild-type (WT) mice was assessed by quantitative real-time polymerase chain reaction ( $\mathrm{n}=4$ for each group). * p-value from unpaired Student's t test was used comparing transgenic vs. WT mice.

(b-c) Expression of miR-195a-5p in hippocampus and temporal cortex of 6-month-old P301S mice compared to WT mice; no significant differences were identified.

(d) FISH images of miR-195a-5p which is stained green in EC of 6-month-old P301S and WT mice. The nuclei were stained blue with DAPI. Cabl1 (stained in red) was used as a marker of EC layer II. Scale bar indicates $20 \mu \mathrm{m} . \mathrm{n}=3$ mice in each group.

(e-h) Analogous experiments comparing EC (e), hippocampus (f) and temporal (g) cortex and performing FISH staining (h) in 6-month-old APP/PS1 mice compared to WT mice. 
Table 1. Overview of the brain samples analyzed in this study.

\begin{tabular}{|c|c|c|c|c|c|c|c|c|c|c|c|c|}
\hline \multirow{2}{*}{ Group } & \multirow{2}{*}{$\begin{array}{c}\text { Total } \\
\text { number }\end{array}$} & \multirow{2}{*}{$\begin{array}{l}\text { Sex } \\
(\mathrm{m} / \mathrm{f})\end{array}$} & \multirow{2}{*}{$\begin{array}{l}\text { Age } \\
( \pm \mathrm{SD})\end{array}$} & \multirow{2}{*}{$\begin{array}{l}\text { Age } \\
\text { range }\end{array}$} & \multirow{2}{*}{$\begin{array}{l}\text { PMI range } \\
\text { (h) }\end{array}$} & \multirow{2}{*}{$\begin{array}{l}\text { RIN value } \\
\text { (range) }\end{array}$} & \multirow{2}{*}{$\begin{array}{l}\text { RNA A260/280 } \\
\text { (range) }\end{array}$} & \multicolumn{5}{|c|}{ Braak Stage } \\
\hline & & & & & & & & 0 & $\mathrm{I} / \mathrm{II}$ & III/ IV & V/VI & n.a. \\
\hline AD cases & 100 & $50 / 50$ & $81.6( \pm 8)$ & $61-95$ & $9-140$ & $3.02(1.2-7.8)$ & $1.90(1.8-1.99)$ & 0 & 0 & 8 & 92 & 0 \\
\hline Controls & 99 & $56 / 43$ & $78.0( \pm 13.5)$ & $41-100$ & $5-168$ & $4.19(2.1-7.6)$ & $1.92(1.8-2.0)$ & 4 & 69 & 13 & 0 & 13 \\
\hline
\end{tabular}

Numbers are for samples from STG analyzed by qPCR. While numbers for EC are slightly lower (AD = 91, controls = 92), the distribution of demographic and clinical variables is essentially identical. n.a. - not available. 
Table 2. Results of targeted differential expression analysis in brain tissue in Alzheimer's disease patients and controls, comparison to previously reported meta-analysis results and results of updated meta-analysis.

\begin{tabular}{|c|c|c|c|c|c|c|c|c|c|c|c|c|c|c|c|}
\hline \multirow{2}{*}{$\begin{array}{l}\text { miR name } \\
\text { (miRBASE) }\end{array}$} & \multicolumn{3}{|c|}{ Current study, STG } & \multicolumn{4}{|c|}{ Current study, EC } & \multicolumn{4}{|c|}{ Meta-analysis (Takousis et al.) } & \multicolumn{4}{|c|}{ Current study, Meta-analysis } \\
\hline & Direction & P-value ${ }^{\S}$ & $\mathrm{N}$ (cases, ctrls) & Direction & P-value ${ }^{\S}$ & Cases & Ctrls & Direction & P-value & $\mathrm{N}$ (cases, ctrls) & $\begin{array}{c}\mathrm{N} \\
\text { studies }\end{array}$ & Direction & P-value & $\mathrm{N}$ (cases, ctrls) & $\begin{array}{c}\mathrm{N} \\
\text { studies }\end{array}$ \\
\hline hsa-miR-125b-5p & down & 0.65 & $186(94,92)$ & up & 0.70 & 90 & 91 & up & 4.13E-13 & $122(64,58)$ & 11 & up & 2.88E-05 & $308(158,150)$ & 12 \\
\hline hsa-miR-501-3p & up & 0.78 & $170(84,86)$ & up & 0.028 & 90 & 91 & up & 2.03E-11 & $68(38,30)$ & 4 & up & $1.55 \mathrm{E}-04$ & $238(122,116)$ & 5 \\
\hline hsa-miR-132-5p & down & $6.90 \mathrm{E}-22$ & $186(94,92)$ & down & 8.3E-05 & 90 & 91 & down & 3.01E-10 & $57(27,30)$ & 5 & down & 2.06E-30 & $243(121,122)$ & 6 \\
\hline hsa-miR-195-5p & up & 0.14 & $186(94,92)$ & up & 0.00014 & 90 & 91 & up & 3.74E-07 & $177(104,73)$ & 7 & up & $4.80 \mathrm{E}-06^{*}$ & $363(198,165)$ & 8 \\
\hline hsa-miR-129-5p & down & $6.00 \mathrm{E}-09$ & $186(94,92)$ & down & $1.40 \mathrm{E}-09$ & 90 & 91 & down & 3.84E-07 & $166(100,66)$ & 6 & down & $1.57 \mathrm{E}-16$ & $1052(621,431)$ & 8 \\
\hline
\end{tabular}

$\S$ - one-sided p-values; \# - two-sided p-values; Ctrls - controls; Bold- differential expression reached significance threshold for the corresponding analysis $(\alpha$ (one-sided) $=0.0167$

for differential expression analyses, $\alpha$ (two-sided) $=1.08 \mathrm{E}-04$ for both meta-analyses; see Methods); *The equivalent P-value after meta-analysis including the EC instead of STG

data is $3.63 \mathrm{E}-10$. 